TITLE:

\title{
A pathophysiological role of TRPV1 in ischemic injury after transient focal cerebral ischemia in mice
}

\section{AUTHOR(S):}

Miyanohara, Jun; Shirakawa, Hisashi; Sanpei, Kazuaki; Nakagawa, Takayuki; Kaneko, Shuji

\section{CITATION:}

Miyanohara, Jun ...[et al]. A pathophysiological role of TRPV1 in ischemic injury after transient focal cerebral ischemia in mice. Biochemical and Biophysical Research Communications 2015, 467(3): 478-483

\section{ISSUE DATE:}

2015-11-20

\section{URL:}

http://hdl.handle.net/2433/241624

\section{RIGHT:}

(c) 2015. This manuscript version is made available under the CC-BY-NC-ND 4.0 license http://creativecommons.org/licenses/by-nc-nd/4.0/.; この論文は出版社版でありません 。引用の際には出版社版をご確認ご利用ください。; This is not the published version. Please cite only the published version. 


\section{A pathophysiological role of TRPV1 in ischemic injury after transient focal cerebral ischemia in mice}

Jun Miyanohara, ${ }^{1}$ Hisashi Shirakawa, ${ }^{1}$ Kazuaki Sanpei, ${ }^{1}$ Takayuki Nakagawa, ${ }^{1,2}$ and Shuji Kaneko $^{1}$

${ }^{1}$ Department of Molecular Pharmacology, Graduate School of Pharmaceutical Sciences, Kyoto University

${ }^{2}$ Department of Clinical Pharmacology and Therapeutics, Kyoto University Hospital

Correspondence to:

Hisashi Shirakawa, PhD, 46-29 Yoshida-shimoadachi-cho, Sakyo-ku, Kyoto, 606-8501, Japan, Tel: +81-75-753-4548; Fax: +81-75-753-4548; E-mail; shirakaw@pharm.kyoto-u.ac.jp

Six keywords:

TRPV1; Knockout mouse; Middle cerebral artery occlusion; Ischemic injury; Capsazepine. 


\section{ABSTRACT}

Transient receptor potential vanilloid 1 (TRPV1) is a non-selective cation channel with high $\mathrm{Ca}^{2+}$ permeability, which functions as a polymodal nociceptor activated by heat, protons and several vanilloids, including capsaicin and anandamide. Although TRPV1 channels are widely distributed in the mammalian brain, their pathophysiological roles in the brain remain to be elucidated. In this study, we investigated whether TRPV1 is involved in cerebral ischemic injury using a middle cerebral artery (MCA) occlusion model in wild-type (WT) and TRPV1-knockout (KO) mice. For transient ischemia, the left MCA of C57BL/6 mice was occluded for 60 min and reperfused at 1 and 2 days after ischemia. We found that neurological and motor deficits, and infarct volumes in TRPV1-KO mice were lower than those of WT mice. Consistent with these results, intracerebroventricular injection of a TRPV1 antagonist, capsazepine (20 nmol), $30 \mathrm{~min}$ before the onset of ischemia attenuated neurological and motor deficits and improved infarct size without influencing cerebral blood flow in the occluded MCA territory. The protective effect of capsazepine on ischemic brain damage was not observed in TRPV1-KO mice. WT and TRPV1-KO mice did not show any differences with respect to the increased number of Iba1-positive microglia/macrophages, GFAP-positive astrocytes, and Gr1-positive neutrophils at 1 and 2 days after cerebral ischemia. Taken together, we conclude that brain TRPV1 channels are activated by ischemic stroke and cause neurological and motor deficits and infarction after brain ischemia.

\section{INTRODUCTION}

Stroke is the second most common cause of death worldwide [1] and the leading cause of adult disability [2]. The burden of stroke is predicted to increase greatly with the anticipated increase in the aging population, which will increase further the demand for new therapies [2]. In fact, current therapeutic approaches, using antiplatelet and thrombolytic agents, only partially ameliorate the clinical outcome of stroke patients since these drugs are not aimed at counteracting the mechanisms associated with neuronal cell death, but at preserving or restoring cerebral blood flow [3].

Transient Receptor Potential Vanilloid 1 (TRPV1), one of the best characterized members of the TRP channel family, is a capsaicin receptor (also known as vanilloid receptor VR1) and is 
activated not only by capsaicin but also by heat $\left(>43^{\circ} \mathrm{C}\right)$, acid $(\mathrm{pH}<6.0)$, and various lipids, i.e., unsaturated N-acyl-dopamines, lipoxygenase products from arachidonic acid, an endocannabinoid anandamide, 2-arachidonoyl-glycerol, and omega-3 polyunsaturated fatty acids [4]. Although TRPV1 is involved in peripheral nociception, TRPV1 is widely expressed in the rodent brain, including the hippocampus, cortex, cerebellum, olfactory bulb, mesencephalon, and hindbrain [5, 6], suggesting that TRPV1 has a physiological role in the CNS. In this context, stimulation of TRPV1 in the ventral midbrain selectively accelerates glutamatergic inputs to midbrain dopaminergic neurons [7]. Moreover, recent research shows that TRPV1 is involved in synaptic plasticity in hippocampal interneurons [8], dentate gyrus [9], and nucleus accumbens [10]. Despite evidence suggesting that TRPV1 has a physiological role in the CNS, little is known about its pathophysiological role in neurological disorders, especially in cerebral ischemic injury.

Therefore, to investigate the possible involvement of TRPV1 in neuronal damage after cerebral ischemia in vivo, we evaluated the effects of TRPV1 deficiency and blockade on infarcted brain volume and neurological and motor function using a mouse model of transient focal cerebral ischemic stroke.

\section{MATERIALS AND METHODS}

\section{Animals}

Male TRPV1-KO and WT mice at 8 weeks of age weighing 20-30 g were used in this study. TRPV1-KO mice were originally provided by Dr Julius and were maintained in our laboratory at a constant ambient temperature of $22 \pm 1^{\circ} \mathrm{C}$ under a 12-h light/dark cycle, and were fed water and chow ad libitum. The experiments were conducted in accordance with the ethical guidelines of Kyoto University Animal Experimentation Committee and the Guidelines of the Japanese Pharmacological Society.

\section{Transient middle cerebral artery (MCA) occlusion}

Mice were anesthetized with $1 \%$ halothane in $30 \% \mathrm{O}_{2}$ and $70 \% \mathrm{~N}_{2} \mathrm{O}$ using a face mask. After a midline skin incision, the left common carotid artery and external artery were isolated and ligated. An 8-0 nylon monofilament coated with silicon (180-200 $\mathrm{mm}$ in diameter) was introduced into the left internal carotid artery through the common carotid artery to occlude the origin of the MCA. After 60 min of occlusion, the monofilament was withdrawn to allow 
reperfusion and the mouse was placed in a thermally controlled incubator $\left(32.0^{\circ} \mathrm{C}\right)$ for $2 \mathrm{~h}$ before being returned to its cage. Rectal temperature was maintained at $37.0 \pm 0.5^{\circ} \mathrm{C}$ with a thermostat-controlled heating pad throughout the operation. Regional cerebral blood flow (rCBF) in the MCA territory was monitored by Laser Dopplar Flowmetry (Omegaflow; Omegawave Inc., Tokyo, Japan). A flexible probe was fixed to the skull (2 $\mathrm{mm}$ posterior and 6 $\mathrm{mm}$ lateral to bregma) before MCA occlusion. Only mice showing $<20 \%$ of baseline rCBF in the MCA territory after occlusion and $>60 \%$ of baseline after reperfusion were used in subsequent experiments.

\section{Intracerebroventricular injection}

The TRPV1 antagonist capsazepine was injected $1 \mathrm{~mm}$ posterior and $1 \mathrm{~mm}$ lateral to the bregma on the left side. A 23-G needle attached to a Hamilton microsyringe was inserted so that its tip was located $3 \mathrm{~mm}$ beneath the dural surface. The needle was kept in the same position for $1 \mathrm{~min}$ before removal. Capsazepine was dissolved in 20\% DMSO/80\% phosphate-buffered saline (PBS) and administered at a dose of $20 \mathrm{nmol} / 5 \mu \mathrm{l}$ per mouse.

\section{Neurological deficit score}

Neurological deficiency was evaluated using a 5-point neurological deficit score test. After several days of ischemia, mice were tested for behavioral changes and scored as follows: 0 , no deficit; 1 , flexion of contralateral forelimb and torso upon lifting the animal by the tail; 2 , circling to the ipsilateral side but normal posture at rest; 3 , circling to the ipsilateral side; 4 , rolling to the ipsilateral side; and 5, no spontaneous motor activity.

\section{Rope grip test}

Motor dysfunction was evaluated by using the 5-point rope grip test. After several days of ischemia, mice were tested for behavioral changes and scored as follows: 0 , escape; 1 , grasping the tightrope with the four legs and the tail; 2 , hanging from the forelimbs and the hindlimbs; 3 , hanging from the forelimbs; 4, trying to hang from the forelimbs; and 5, fall. The final score was the average of five trials.

\section{Measurement of infarct volume}

After neurological deficit score evaluation, mice were sacrificed, the brains were removed, and the forebrains were sliced into eight coronal 1-mm sections using a mouse brain matrix. The 
slices were placed in $2 \%$ 2,3,5-triphenyltetrazolium chloride (TTC) at $37^{\circ} \mathrm{C}$ for $20 \mathrm{~min}$. The infarct area of each brain slice was quantified using image analysis software (Image J). The infarct volumes were calculated by multiplying each area by the slice thickness.

\section{Immunohistochemistry and Immunofluorescence}

Mice were intraperitoneally injected with $50 \mathrm{mg} / \mathrm{kg}$ pentobarbital and perfused transcardially with $0.1 \mathrm{M}$ PBS followed by $4 \%$ paraformaldehyde in $0.1 \mathrm{M}$ PBS. Brains were stored in fixative for $3 \mathrm{~h}$ and then transferred into $0.1 \mathrm{M}$ PBS containing 15\% sucrose for $24 \mathrm{~h}$. Coronal sections with a $20 \mu \mathrm{m}$ thickness were obtained using a cryomicrotome (Leica, Bensheim, Germany). Brain sections were soaked in $0.1 \%$ Triton X100 for permeabilization and then incubated overnight at $4^{\circ} \mathrm{C}$ with the following primary antibodies: a rabbit polyclonal anti-Iba1 antibody (1:500 dilution, Wako); a rabbit polyclonal anti-GFAP antibody (1:1000 dilution, DAKO); and a rat monoclonal anti-Gr1 (Ly6G) antibody (1:300 dilution, R\&D Systems). For immunofluorescence, sections were incubated with secondary antibodies (Alexa Flour 594-labeled donkey anti-rabbit IgG (1:300 dilution, Molecular Probes) and Alexa Flour 488-labeled donkey anti-rat IgG (1:300 dilution, Molecular Probes)) for $1.5 \mathrm{~h}$ in the dark at room temperature. Immunofluorescence was visualized with an Olympus FluoView microscope equipped with a laser scanning confocal imaging system. The number of Iba1- and GFAP-immunoreactive cells in the peri-ischemic area was counted in five $500 \times 500 \mu \mathrm{m}^{2}$ fields at approximately $+0.7 \mathrm{~mm}$ from bregma. The number of Gr1-positive cells in the ischemic core area was counted in five $500 \times 500 \mu \mathrm{m}^{2}$ fields at approximately $+0.7 \mathrm{~mm}$ from bregma.

\section{Data analysis}

Data are presented as the mean \pm SEM and were analyzed using GraphPad Prism version 5.0. Neurological deficit scores, results of the rope grip test, and infarct volumes were analyzed using Student's t-test. The numbers of $\mathrm{Iba}^{+}$or $\mathrm{GFAP}^{+}$cells were analyzed by two-way analysis of variance (ANOVA) followed by the post hoc Bonferroni test. In all cases, differences of $p<$ 0.05 were considered statistically significant.

\section{RESULTS}

TRPV1 gene deficiency improves neurological outcome and reduces infarct volume 
To investigate the involvement of TRPV1 in ischemic-reperfusion injury, we examined the effects of TRPV1 deficiency on ischemic brain injury using a mouse focal ischemia model. The left MCA was occluded for 60 min followed by reperfusion. MCA occlusion in WT and TRPV1-KO mice decreased rCBF to $<20 \%$ of baseline and reperfusion restored rCBF. No differences in rCBF were observed between the two mouse genotypes (Fig. 1A). Neurological deficiency levels were similar between WT and TRPV1-KO mice at 1 day after ischemia; however, at 2 days after reperfusion, TRPV1-KO mice showed less neurological deficiency than WT mice (Fig. 1B). Motor dysfunction was less severe in TRPV1-KO mice than in WT mice at 1 and 2 days after reperfusion (Fig. 1C). Infarct volume was evaluated by TTC staining after performing the behavioral test. The volume in TRPV1-KO mice was significantly lower than that in WT mice at 2 days after ischemia. (Fig. 1D and E).

\section{Protective effect of capsazepine on neurological deficit and infarct volume}

Gene inactivation is known to result in some cases in the activation of compensatory or redundancy mechanisms that counteract the loss of gene function. Therefore, we investigated the effects of the TRPV1 antagonist capsazepine on ischemic brain injury. Thirty minutes after intracerebroventricular injection of vehicle or capsazepine (20 nmol), no differences in rCBF were observed between either group of mice (Fig. 2A). At 2 days after reperfusion, the degree of neurological deficiency and motor dysfunction in capsazepine-treated mice was significantly lower than that in vehicle-treated mice (Fig. 2B and C). In addition, the infarct volume was significantly smaller in capsazepine-treated mice than in vehicle-treated mice (Fig. 2D and E).

\section{No capsazepine protective effect in TRPV1-KO mice after ischemic brain injury}

To confirm that the protective effect of capsazepine was TRPV1-specific, we examined the effect of capsazepine in TRPV1-KO mice. rCBF was unaffected when vehicle or capsazepine was injected into TRPV1-KO mice at $30 \mathrm{~min}$ before MCA occlusion (Fig. 3A). Additionally, capsazepine had no significant effects on neurological deficiency, motor dysfunction, or infarct volume in TRPV1-KO (Figs. 3B, 3C, 3D, and 3E), indicating that the protective effects of capsazepine require TRPV1.

\section{The effect of TRPV1 deficiency on microglia/macrophages, astrocyte, and neutrophil accumulation}


Finally, we examined accumulation of microglia/macrophages and astrocytes in the peri-ischemic area and of neutrophils in the ischemic core area at 1 and 2 days after ischemia by immunohistochemical staining for Iba1, GFAP, and Gr1, respectively. The numbers of Iba1-positive microglia/macrophages (Fig. 4A), GFAP-positive astrocytes (Fig. 4B), and Gr1-positive neutrophils (Fig. 4C) were higher at 1 and 2 days after cerebral ischemia; however, there were no differences between TRPV1-KO and WT mice with respect to the increases in the numbers of each cell type.

\section{DISCUSSION}

The present study clearly demonstrated that TRPV1 gene knockout attenuates neurological deficiency and motor dysfunction and reduces infarct volume after cerebral ischemia. Moreover, intracerebroventricular injection of capsazepine improved neurological outcome and reduced infarct volume in WT mice, but not in TRPV1-KO mice. These results suggest strongly that TRPV1 activation has a deleterious effect on the pathophysiology of brain ischemia.

TRPV1 is expressed in a variety of cell types in both the peripheral and central nervous system [5, 6], suggesting that it functions in several cell populations to increase the deleterious effects of ischemic brain injury. In this study, however, a single injection of capsazepine into the brain was as effective as global gene deletion of the TRPV1 gene in attenuating neurological deficit and motor dysfunction, and reduced infarct volume after ischemic brain injury, indicating that TRPV1 function at least affects recovery from ischemic brain injury. Previous reports indicate that TRPV1 is expressed in microglia [11, 12] and astrocytes [13] in the brain. In the present study, the number of Iba1-immunopositive microglia/macrophages, GFAP-immunopositive astrocytes Gr1-immunopositive neutrophils increased at 1 and 2 days after cerebral ischemia; however, no differences were observed between WT and TRPV1-KO mice, suggesting that TRPV1 in these cells is not critical for cerebral ischemic injury.

To date, electrophysiological studies clearly demonstrate that TRPV1 activation modifies synaptic transmission via both presynaptic $[7,8,14-16]$ and postsynaptic mechanisms [9, 10]. Stimulation of TRPV1 by capsaicin or an endogenous TRPV1 agonist anadamide increased the frequency of spontaneous excitatory postsynaptic currents in dopaminergic neurons of the substantia nigra pars compacta [7], striatal neurons [14] and subpopulations of periaqueductal gray neurons [15]. Moreover, Shoudai et al. [16] reported that $\mathrm{Ca}^{2+}$ entry through TRPV1 channels triggered tonically 
glutamate release independently of voltage-activated calcium channels in the central terminals of peripheral nerves. These studies clearly indicate that TRPV1 stimulation potentiates glutamatergic release in the CNS. Considering that glutamate is not only the primary excitatory neurotransmitter in the adult brain, but also a critical transmitter for the degeneration of signaling neurons following stroke [17], and that the levels of the TRPV1 endogenous agonists, palmitoylethanolamide [20] and anandamide [21], in the brain are elevated in cerebral ischemia, it is conceivable that atypical and excessive activation of TRPV1 during cerebral ischemia leads to the pathophysiological deterioration caused by neurotoxicity. Additionally, our previous data show endogenous stimulation of TRPV1-triggered neurotoxicity in cultured rat cortical neurons [18], indicating that TRPV1-mediated somatodendritic or postsynaptic $\mathrm{Ca}^{2+}$ influx could directly affect neuronal survival. If the expression level of TRPV1 is upregulated in the CNS neurons of stroke patients, as is previouly reported in mesial temporal lobe epilepsy patients [19], the neurotoxicity mediated by TRPV1would be further enhanced in cerebral ischemia.

Ischemic stroke increases anaerobic glycolysis and lactate accumulation, and decreases $\mathrm{pH}$ (acidosis) [22] in the brain. It also increases the accumulation of various neurotransmitters and inflammatory substances, including as major pain-causing substances bradykinin [23] and prostaglandin [24], which can induce the open state of the multistate TRPV1 receptor via phosphorylation by protein kinase $\mathrm{C}$ (PKC) induced by $\mathrm{G}_{\mathrm{q} / 11}$-coupled receptor activation [4]. Additionally, a recent study revealed that bradykinin-induced PKC phosphorylation causes TRPV1 activation and potentiates glutamatergic synaptic transmission at the first sensory synapse [25]. Taken together, TRPV1 in neurons could be activated under ischemic conditions in response to various stimuli, and this could lead to an increase in neurotoxic glutamate release and adverse effects on neuronal survival.

Although intracerebroventricular injection of the TRPV1 blocker capsazepine attenuated ischemic brain injury, nonspecific effects of capsazepine might be anticipated, such as inhibition of TRPM8 [26]. In this study, however, the protective effect of capsazepine was not evident in TRPV1-KO mice, indicating that the attenuation of the deleterious effects of ischemic brain injury by capsazepine was TRPV1 specific.

By contrast, previous research shows that TRPV1 activation exerts protective effects in a rodent ischemia model, possibly by inducing hypothermia [27, 28]. Although an injection of $25 \mathrm{mg} / \mathrm{kg}$ rinvanil, a TRPV1 agonist, induced hypothermia and exerted neuroprotective effects against cerebral ischemic injury, a higher dose $(50 \mathrm{mg} / \mathrm{kg}$ ) did not show any significant effect [27], indicating that excessive stimulation of TRPV1 can adversely affect neuronal survival. Furthermore, we evaluated 
infarct volume under more severe conditions than those employed in a previous report using dihydrocapsaicin [28]. Therefore, it is possible that a difference in the extent of severity of brain injury explains the difference between our results and those reported previously, although further investigation will be needed to confirm this. In this context, further evidence that peripheral TRPV1 activation is primarily involved in hypothermia comes from studies showing the involvement of constitutive activation of TRPV1 channels by protons in the abdominal viscera in thermoregulation [29] and attenuation of hyperthermia induced by a TRPV1 antagonist, AMG9810, by the $\beta$-adrenoceptor antagonist propranolol [30]. This notion is further reinforced by results showing that the TRPV1 antagonist AS1928370 with high brain penetrability has little effect on hyperthermia [31]. Thus, at low doses of the TRPV1 agonists that do affect neurons in the CNS, hypothermia-dependent ischemic neuroprotection might be the predominant effect. However, judging from the contradictory effects of TRPV1 on ischemia, therapeutic interventions designed to induce hypothermia via TRPV1 activation could entail a risk of CNS neurotoxicity triggered by mistakenly or unexpectedly high dose treatments with TRPV1 agonists. Taken together, selective inhibition of TRPV1 in the CNS with doses of antagonists that do not affect peripheral TRPV1 activity could be more effective in preventing TRPV1-mediated neurotoxicity when used in combination with the well-established cooling system.

In conclusion, we suggest that TRPV1 plays a significant role in ischemic brain injury. TRPV1 could be the promising target for clinical interventions designed to treat ischemic brain injury.

\section{Conflict of interest}

The authors declare no competing financial interests.

\section{Acknowledgements}

This study was supported by a Grant-in-aid for Scientific Research from the Ministry of Education, Culture, Sports, Science, and Technology, Japan, and from the Japan Society for the Promotion of Science. This work was also supported by the Naito Foundation and the Takeda Science Foundation. We thank especially Dr Yasushi Takagi for technical support with the MCA occlusion experiments. 


\section{References}

[1] C.J.L. Murray, A.D. Lopez, Mortality by cause for eight regions of the world: Global Burden of Disease Study. Lancet. 349 (1997) 1269-1276.

[2] G.A. Donnan, M. Fisher, M. Macleod, S.M. Davis, Stroke. Lancet. 371 (2008) 1612-1623.

[3] P. Deb, S. Sharma, K.M. Hassan, Pathophysiologic mechanisms of acute ischemic stroke: An overview with emphasis on therapeutic significance beyond thrombolysis. Pathophysiology 17 (2010) 197-218.

[4] M. Tominaga, T. Tominaga, Structure and function of TRPV1. Pflugers Arch. 451 (2005) 143-150.

[5] A. Tóth, J. Boczan, N. Kedei, E. Lizanecz, Z. Bagi, Z. Papp, I. Edes, L. Csiba, P.M. Blumberg, Expression and distribution of vanilloid receptor 1 (TRPV1) in the adult rat brain. Brain Res. Mol. Brain Res. 135 (2005) 162-168.

[6] D. Martins, I. Tavares, C. Morgado, "Hotheaded": the role OF TRPV1 in brain functions. Neuropharmacology 85 (2014) 151-157.

[7] S. Marinelli, V. Di Marzo, N. Berretta, I. Matias, M. Maccarrone, G. Bernardi, N.B. Mercuri, Presynaptic facilitation of glutamatergic synapses to dopaminergic neurons of the rat substantia nigra by endogenous stimulation of vanilloid receptors. J. Neurosci. 23 (2003) 3136-3144.

[8] H.E. Gibson, J.G. Edwards, R.S. Page, M.J. Van Hook, J.A. Kauer, TRPV1 channels mediate long-term depression at synapses on hippocampal interneurons. Neuron 57 (2008) 746-759.

[9] A.E. Chávez, C.Q. Chiu, P.E. Castillo, TRPV1 activation by endogenous anandamide triggers postsynaptic long-term depression in dentate gyrus. Nat. Neurosci. 13 (2010) 1511-1518.

[10] B.A. Grueter, G. Brasnjo, R.C. Malenka, Postsynaptic TRPV1 triggers cell type-specific long-term depression in the nucleus accumbens. Nat. Neurosci. 13 (2010) 1519-1525.

[11] S.R. Kim, S.U. Kim, U. Oh, B.K. Jin, Transient Receptor Potential Vanilloid Subtype 1 Mediates Microglial Cell Death In Vivo and In Vitro via Ca2+-Mediated Mitochondrial Damage and Cytochrome c Release. J. Immunol. 177 (2006) 4322-4329. 
[12] T. Miyake, H. Shirakawa, T. Nakagawa, S. Kaneko, Activation of mitochondrial transient receptor potential vanilloid 1 channel contributes to microglial migration. Glia 63 (2015) 1870-1882.

[13] T. Mannari, S. Morita, E. Furube, M. Tominaga, S. Miyata, Astrocytic TRPV1 ion channels detect blood-borne signals in the sensory circumventricular organs of adult mouse brains. Glia 61 (2013) 957-971.

[14] A. Musella, V. De Chiara, S. Rossi, C. Prosperetti, G. Bernardi, M. Maccarrone, D. Centonze, TRPV1 channels facilitate glutamate transmission in the striatum. Mol. Cell Neurosci. 40 (2009) 89-97.

[15] H. Kawahara, G.M. Drew, M.J. Christie, C.W. Vaughan, Inhibition of fatty acid amide hydrolase unmasks CB1 receptor and TRPV1 channel-mediated modulation of glutamatergic synaptic transmission in midbrain periaqueductal grey. Br. J. Pharmacol. 163 (2011) 1214-1222.

[16] K. Shoudai, J.H. Peters, S.J. McDougall, J.A. Fawley, M.C. Andresen, Thermally active TRPV1 tonically drives central spontaneous glutamate release. J. Neurosci. 30 (2010) 14470-14475.

[17] T.W. Lai, S. Zhang, Y.T. Wang, Excitotoxicity and stroke: identifying novel targets for neuroprotection. Prog. Neurobiol. 115 (2014) 157-188.

[18] H. Shirakawa, T. Yamaoka, K. Sanpei, H. Sasaoka, T. Nakagawa, S. Kaneko, TRPV1 stimulation triggers apoptotic cell death of rat cortical neurons. Biochem. Biophys. Res. Commun. 377 (2008) 1211-1215.

[19] F.J. Sun, W. Guo, D.H. Zheng, C.Q. Zhang, S. Li, S.Y. Liu, Q. Yin, H. Yang, H.F. Shu, Increased expression of TRPV1 in the cortex and hippocampus from patients with mesial temporal lobe epilepsy. J. Mol. Neurosci. 49 (2013) 182-193.

[20] A. Franklin, S. Parmentier-Batteur, L. Walter, D.A. Greenberg, N. Stella, Palmitoylethanolamide Increases after Focal Cerebral Ischemia and Potentiates Microglial Cell Motility. J. Neurosci. 23 (2003) 7767-7775.

[21] S. Muthian, D.J. Rademacher, C.T. Roelke, G.J. Gross, C.J. Hillard, Anandamide content is increased and CB1 cannabinoid receptor blockade is protective during transient, focal cerebral ischemia. Neuroscience 129 (2004) 743-750.

[22] N.M. Robbins, R.A. Swanson, Opposing effects of glucose on stroke and reperfusion injury: acidosis, oxidative stress, and energy metabolism. Stroke 45 (2014) 1881-1886.

[23] M. Gröger, D. Lebesgue, D. Pruneau, J. Relton, S.W. Kim, J. Nussberger, N. Plesnila, 
Release of bradykinin and expression of kinin B2 receptors in the brain: role for cell death and brain edema formation after focal cerebral ischemia in mice. J. Cereb. Blood Flow Metab. 25 (2005) 978-989.

[24] T. Kawano, J. Anrather, P. Zhou, L. Park, G. Wang, K.A. Frys, A. Kunz, S. Cho, M. Orio, C. Iadecola, Prostaglandin E2 EP1 receptors: downstream effectors of COX-2 neurotoxicity. Nat. Med. 12 (2006) 225-229.

[25] P. Sikand, L.S. Premkumar, Potentiation of glutamatergic synaptic transmission by protein kinase C-mediated sensitization of TRPV1 at the first sensory synapse. J. Physiol. 581 (2007) 631-647.

[26] H.J. Behrendt, T. Germann, C. Gillen, H. Hatt, R. Jostock, Characterization of the mouse cold-menthol receptor TRPM8 and vanilloid receptor type-1 VR1 using a fluorometric imaging plate reader (FLIPR) assay. Br. J. Pharmacol. 141 (2004) 737-745.

[27] M. Muzzi, R. Felici, L. Cavone, E. Gerace, A. Minassi, G. Appendino, F. Moroni, A. Chiarugi, Ischemic neuroprotection by TRPV1 receptor-induced hypothermia. J. Cereb. Blood Flow Metab. 32 (2012) 978-982.

[28] Z. Cao, A. Balasubramanian, S.P. Marrelli, Pharmacologically induced hypothermia via TRPV1 channel agonism provides neuroprotection following ischemic stroke when initiated 90 min after reperfusion. Am. J. Physiol. Regul. Integr. Comp. Physiol. 306 (2014) R149-156.

[29] A. Garami, Y.P. Shimansky, E. Pakai, D.L. Oliveira, N.R. Gavva, A.A. Romanovsky, Contributions of different modes of TRPV1 activation to TRPV1 antagonist-induced hyperthermia. J. Neurosci. 30 (2010) 1435-1440.

[30] K.M. Alawi, A.A. Aubdool, L. Liang, E. Wilde, A. Vepa, M.P. Psefteli, S.D. Brain, J.E. Keeble, The sympathetic nervous system is controlled by transient receptor potential vanilloid 1 in the regulation of body temperature. FASEB J. (2015).

[31] T. Watabiki, T. Kiso, T. Kuramochi, K. Yonezawa, N. Tsuji, A. Kohara, S. Kakimoto, T. Aoki, N. Matsuoka, Amelioration of neuropathic pain by novel transient receptor potential vanilloid 1 antagonist AS1928370 in rats without hyperthermic effect. J. Pharmacol. Exp. Ther. 336 (2011) 743-750.

\section{FIGURE LEGENDS}


Fig. 1. Attenuation of the neurological and motor deficit and infarct volume in TRPV1-deficient mice. (A) Decreases in rCBF in the MCA territory were estimated by Laser-Doppler Flowmetry. Percent changes from baseline were recorded at baseline, at 5 min after induction of ischemia, and at $10 \mathrm{~min}$ intervals from onset of ischemia to $25 \mathrm{~min}$ after reperfusion, in WT and TRPV1-KO mice. (B) At 1 and 2 days after reperfusion, the neurological deficits of WT and TRPV1-KO mice ( $\mathrm{n}=15-22)$ were assessed. (C) At 1 and 2 days after reperfusion, the motor deficits of WT and TRPV1-KO mice ( $\mathrm{n}=15-22)$ were assessed. (D) Representative images of TTC stained coronal sections obtained from WT and TRPV1-KO mice. (D) Infarct volume at 2 days after reperfusion in WT and TRPV1-KO mice (n $=8-9) .{ }^{*} p<0.05$ vs. WT.

Fig. 2. Protective effects of capsazepine pre-treatment on ischemic brain injury. (A) Decreases in $\mathrm{rCBF}$ in the MCA territory were estimated by Laser-Doppler Flowmetry. Percent changes from baseline were recorded at baseline, at $5 \mathrm{~min}$ after induction of ischemia, and at $10 \mathrm{~min}$ intervals from onset of ischemia to 25 min after reperfusion, in vehicle- and capsazepine- (20 nmol) treated mice. (B) At 1 and 2 days after reperfusion, the neurological deficits of vehicleand capsazepine-treated mice ( $\mathrm{n}=11$, respectively) were assessed. (C) At 1 and 2 days after reperfusion, the motor deficits of vehicle- and capsazepine-treated mice ( $n=11$, respectively) were assessed. (D) Representative images of TTC-stained coronal sections obtained from vehicle- and capsazepine-treated mice. (E) Infarct volume at 2 days after reperfusion in vehicleand capsazepine-treated mice ( $\mathrm{n}=11$, respectively). ${ }^{*} p<0.05$, ${ }^{* *} p<0.01$ vs. vehicle.

Fig. 3. Null effect of capsazepine on infarct volume in TRPV1-deficient mice. (A) Decreases in rCBF in the MCA territory were estimated by Laser-Doppler Flowmetry. Percent changes from baseline were recorded at baseline, at 5 min after induction of ischemia, and at 10 min intervals from onset of ischemia to $25 \mathrm{~min}$ after reperfusion, in vehicle- and capsazepine- (20 nmol) treated TRPV1-KO mice. (B) At 1 and 2 days after reperfusion, the neurological deficits of vehicle- and capsazepine-treated TRPV1-KO mice ( $n=10$, respectively) were assessed. (C) At 1 and 2 days after reperfusion, the motor deficits of vehicle- and capsazepine-treated TRPV1-KO mice ( $\mathrm{n}=10$, respectively) were assessed. (D) Representative images of TTC stained coronal sections obtained from vehicle- and capsazepine-treated TRPV1-KO mice. (E) Infarct volume at 2 days after reperfusion in vehicle- and capsazepine-treated TRPV1-KO mice ( $\mathrm{n}=10$, respectively). 
Fig. 4. Null effect of TRPV1 deficiency on the number of microglia/macrophages, astrocytes and neutrophils after ischemia. The upper inset depicts ischemic core area (core), peri-infarct area (peri), and contralateral area (contra) in an ischemic brain slice. (A) Summarized data of the number of Iba1-positive cells at 1 and 2 days after ischemia (left) and representative images of Iba1-positive cells at 2 days after ischemia (right). (B) Summarized data of the number of GFAP-positive cells at 1 and 2 days after ischemia (left) and representative images of GFAP-positive cells at 2 days after ischemia (right). (C) Summarized data of the number of Gr1-positive cells at 1 and 2 days after ischemia (left) and representative images of Gr1-positive cells at 2 days after ischemia (right). $\mathrm{n}=5-6$, respectively. 
Fig. 1
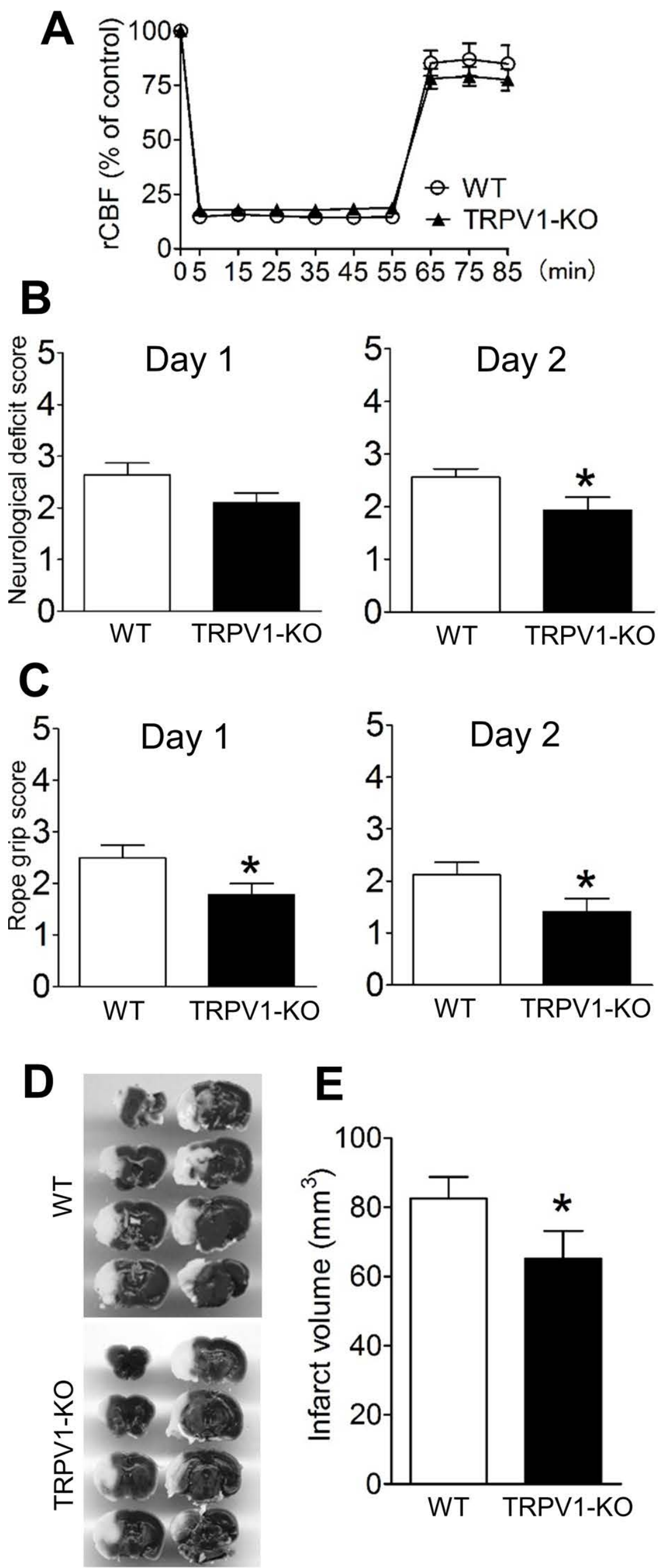

E

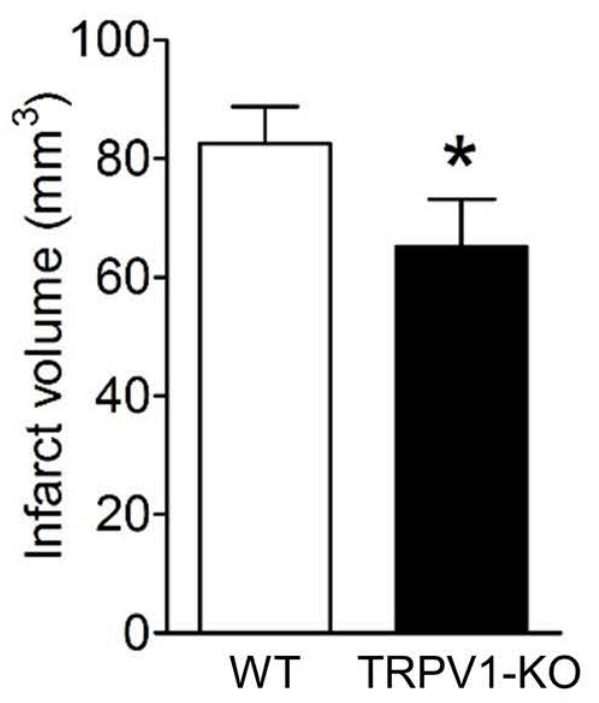


Fig. 2

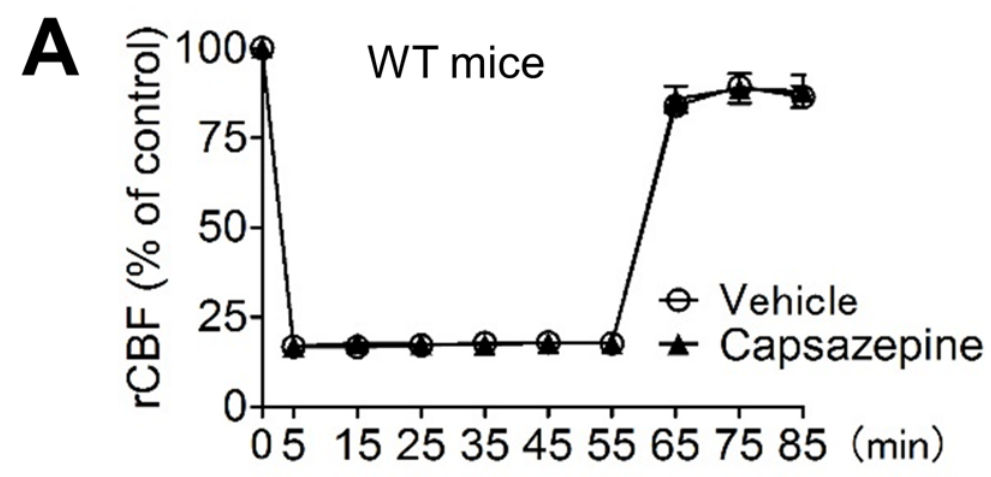

\section{B}
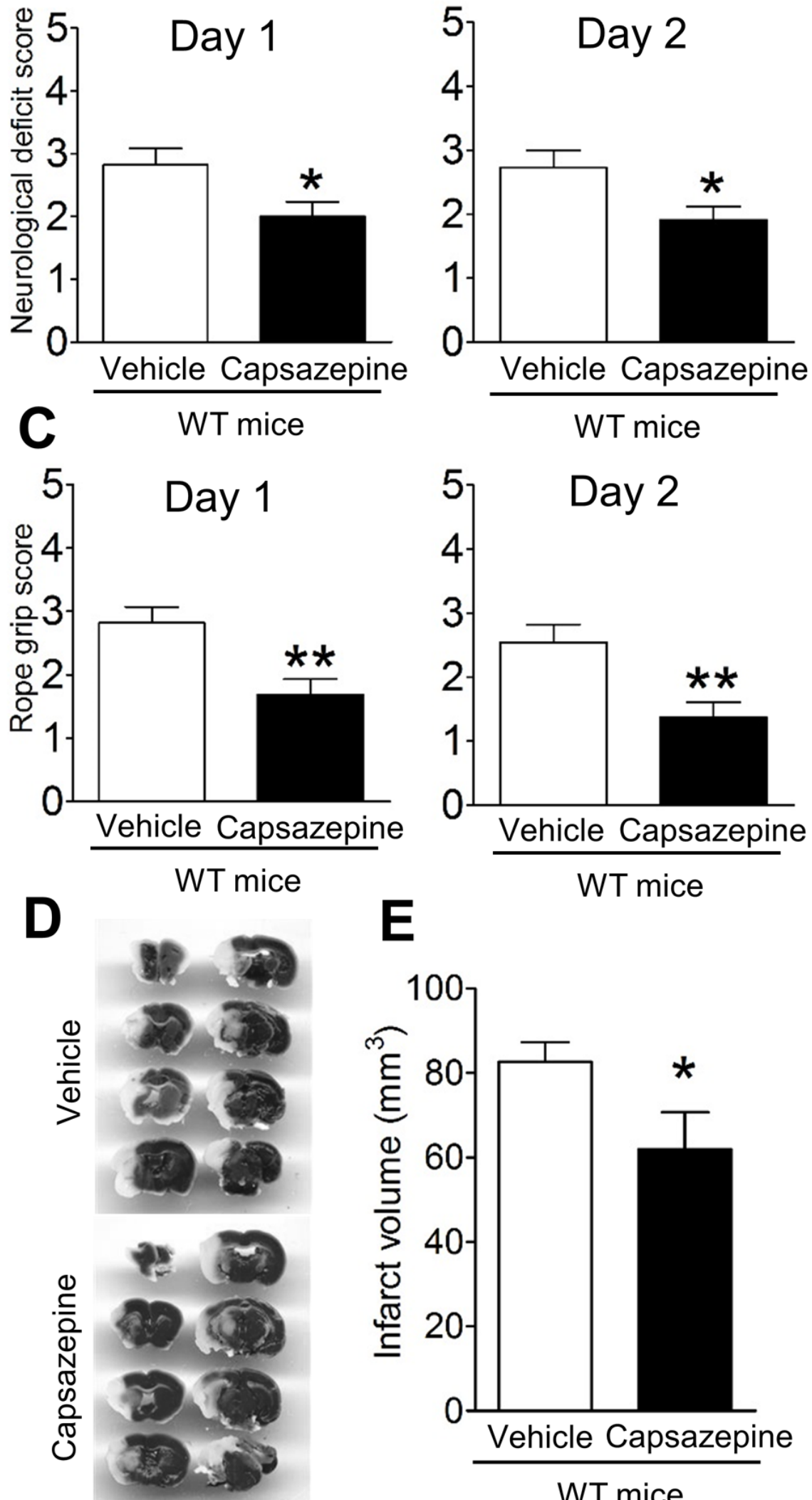

WT mice

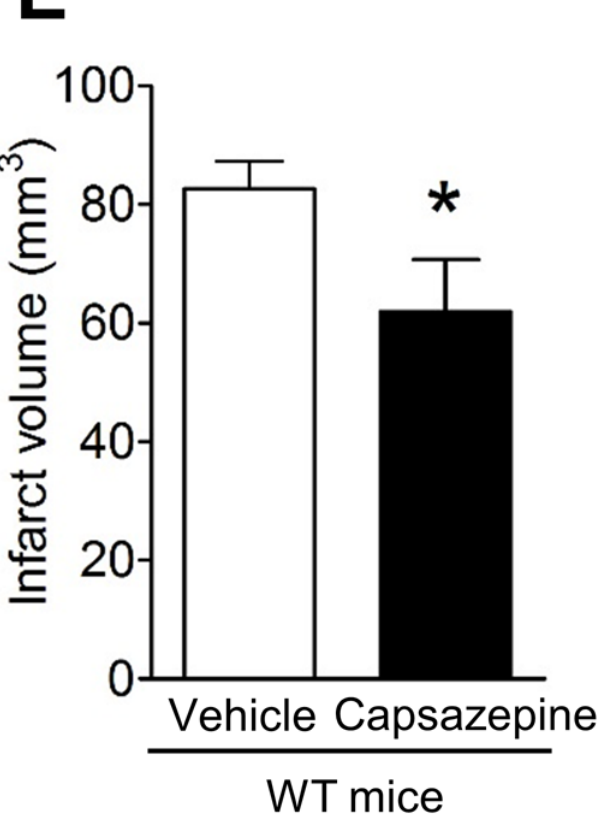


Fig. 3

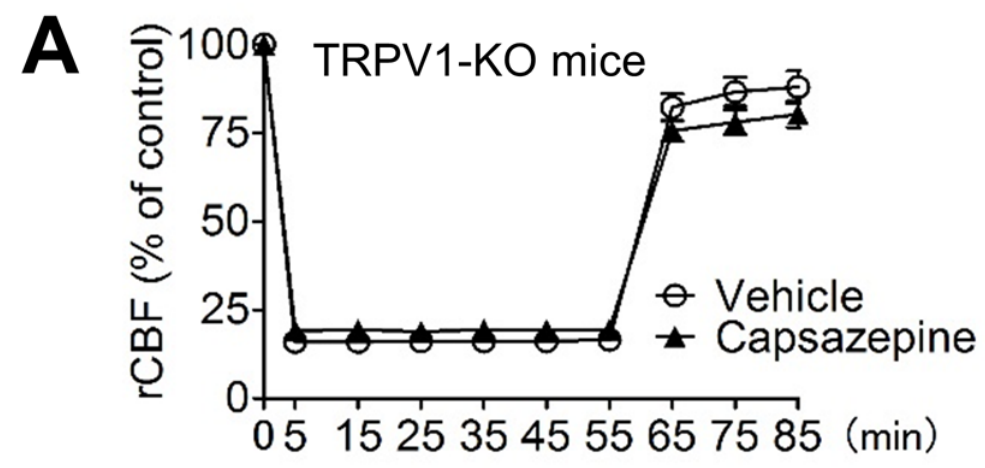

\section{B}
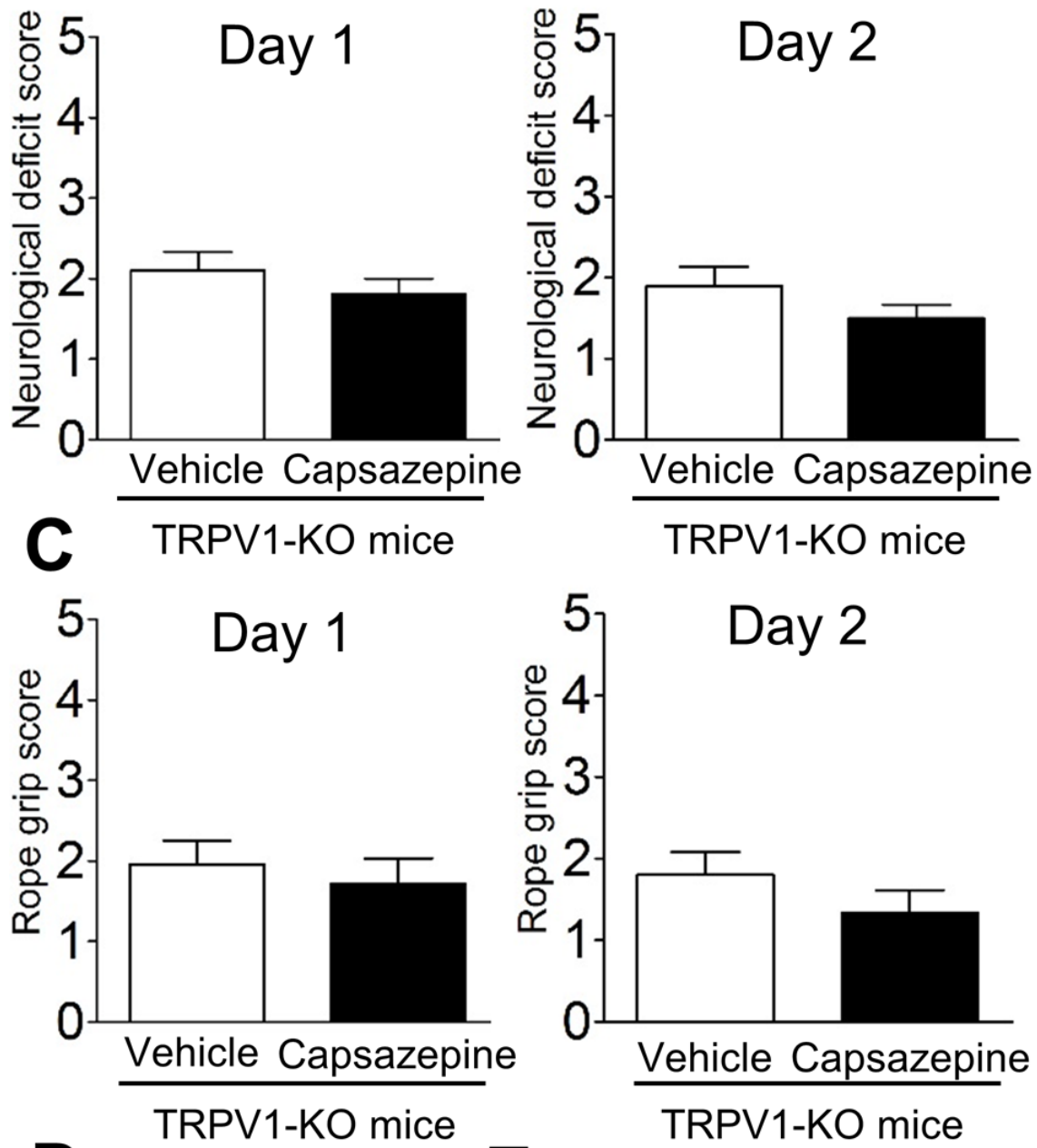

D

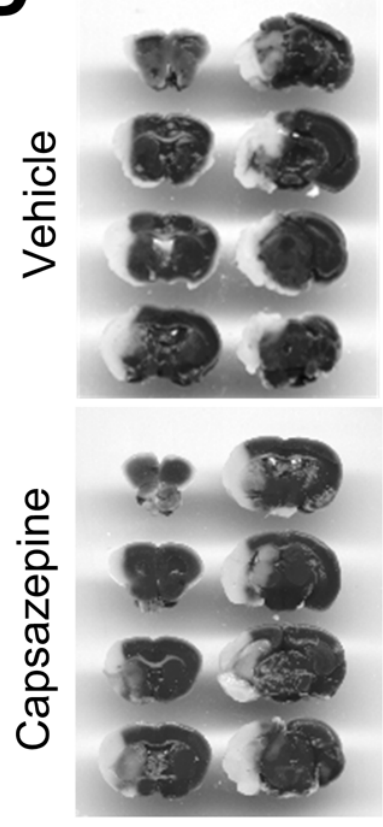

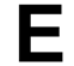

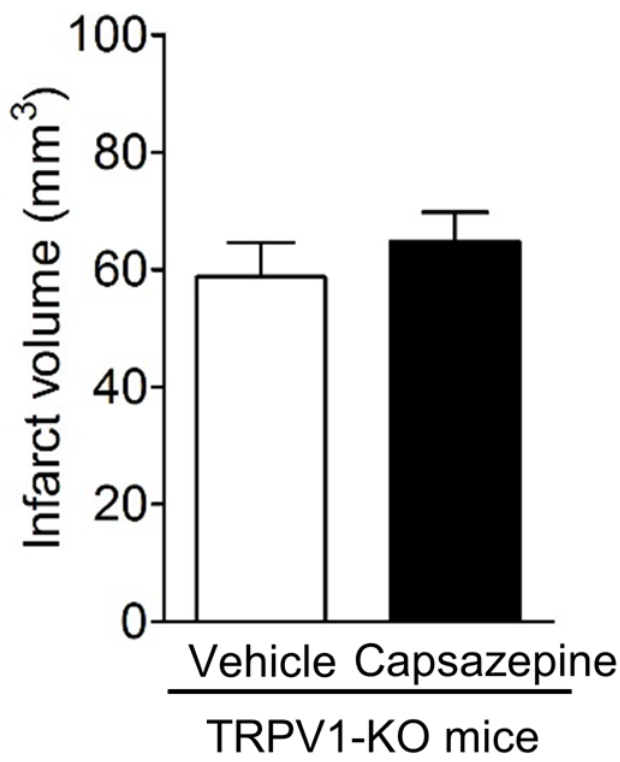


Fig. 4

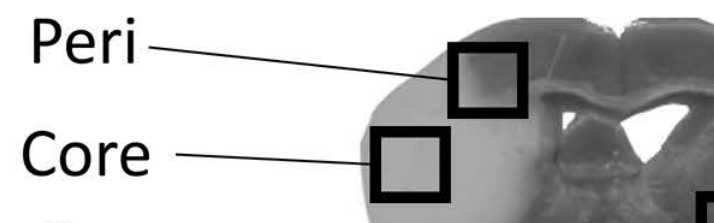

Contra

A

ते

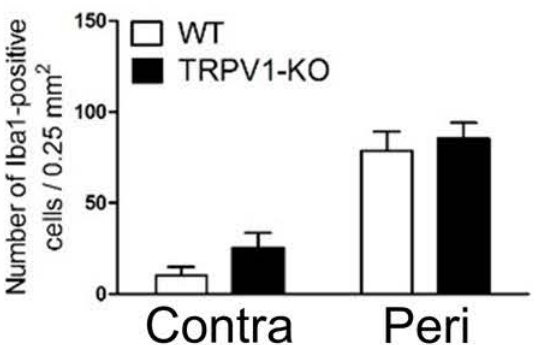

Day 2
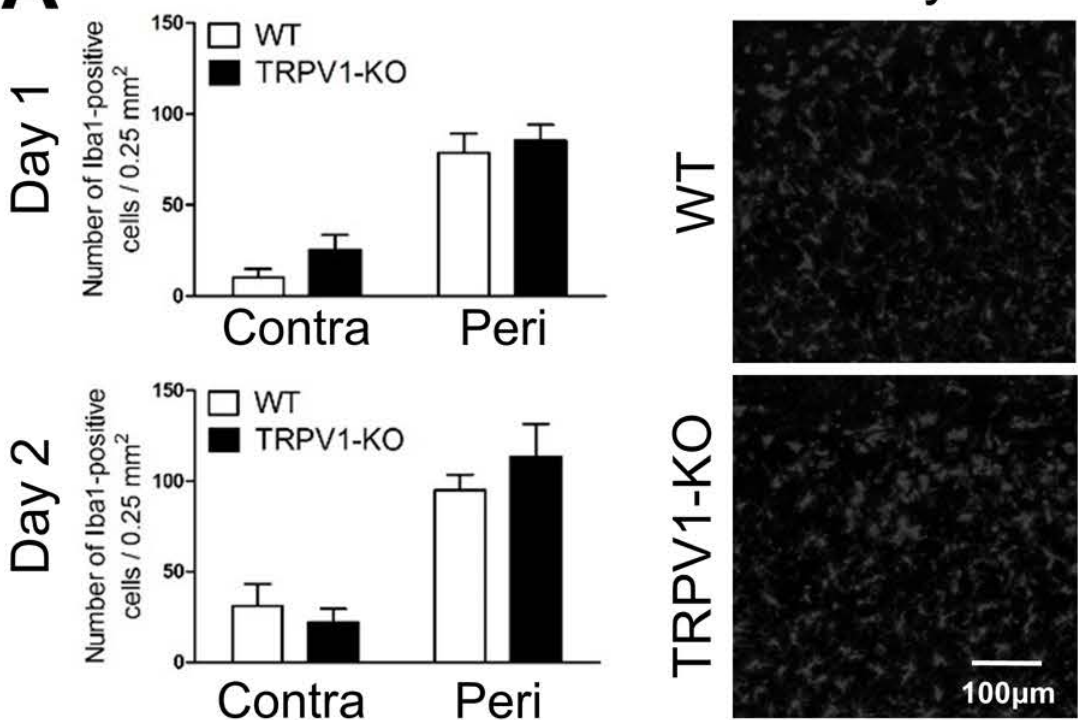

B

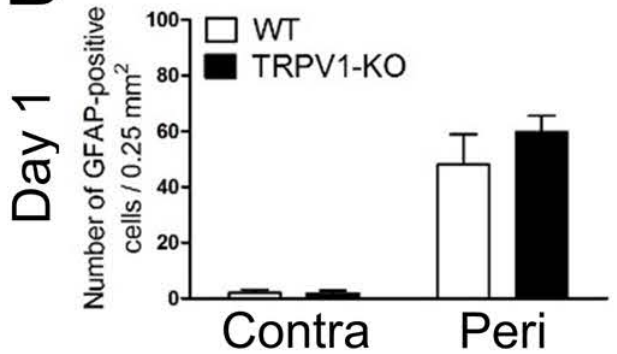

Day 2
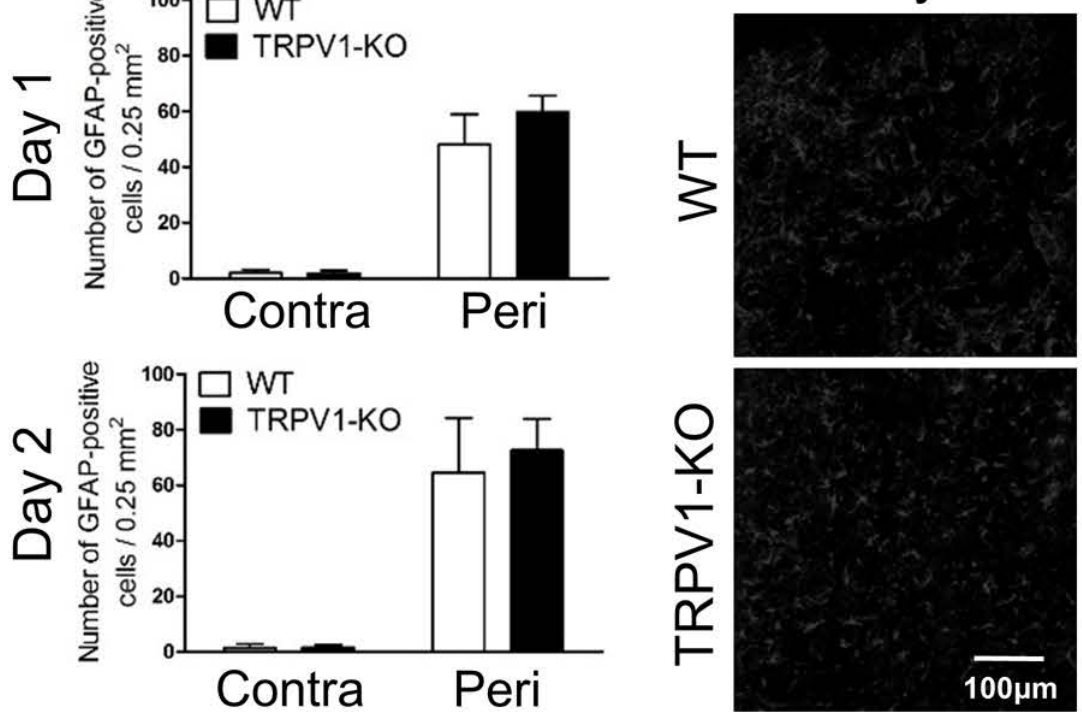

$\mathrm{c}$

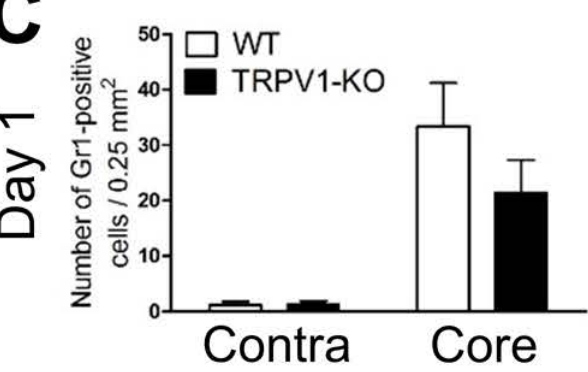

Day 2
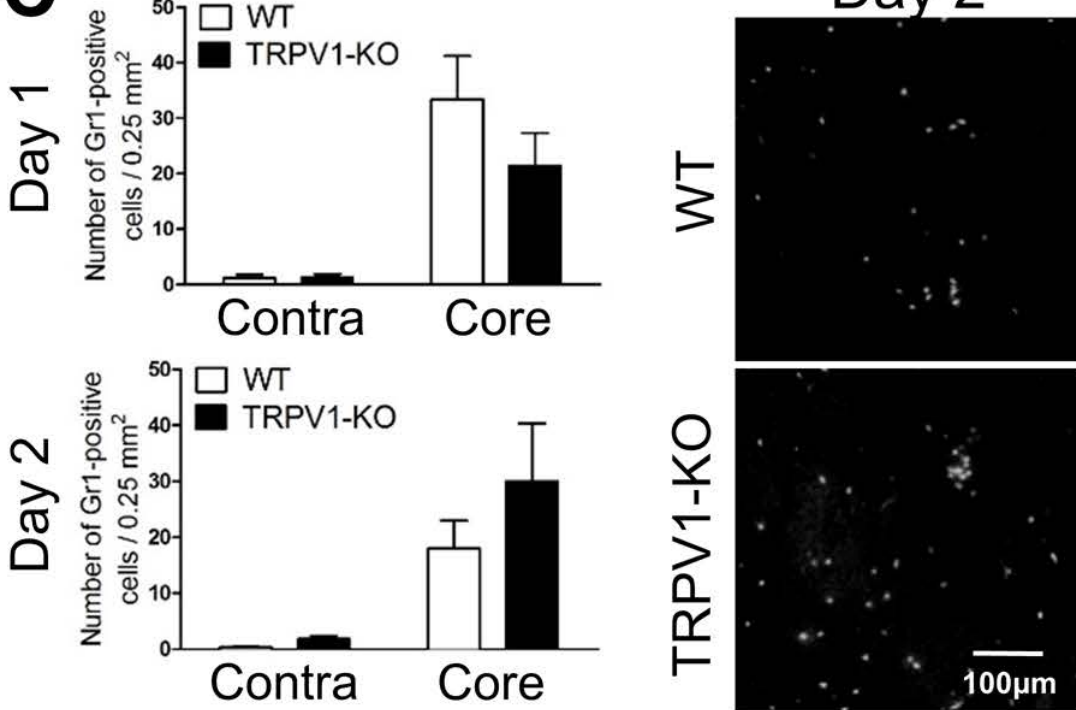